\title{
Indirect contact freeze water desalination for an ice maker machine - CFD simulation
}

\author{
Harith Jayakody ${ }^{1,}$, Raya Al-Dadah ${ }^{1}$, and Saad Mahmoud ${ }^{1}$ \\ ${ }^{1}$ School of Mechanical Engineering, University of Birmingham, Birmingham, B15 2TT, U.K
}

\begin{abstract}
To offer for potable water shortages, sea water desalination is a potential solution for the global rising demand for fresh water. The latent heat of fusion is about one-seventh the latent heat of vaporisation, thus indicating the benefit of lower energy consumption for the freeze desalination process. Limited literature is reported on computational fluid dynamics (CFD) on freeze desalination. Therefore, analysing and investigating thermodynamic processes are easily conducted by the powerful tool of CFD. A single unit of ice formation in an ice maker machine was modelled using ANSYS Fluent software three-dimensionally. Energy, species transport and solidification/melting modules were used in building the CFD model. Parametric analysis was conducted using the established CFD model to predict the effects of freezing temperature and the geometry of the ice maker machine; on ice production and the freezing time. Lower freezing temperatures allowed more ice production and faster freezing. Increasing the diameter and the length of the freezing tube enabled more ice to be produced.
\end{abstract}

\section{Introduction}

Water is a fundamental element that contributes imperatively in the growth of human society [1]. The approximate value of freshwater supply for the entire water source on Earth is $3 \%$, indicating the scarcity of water. Water shortage concerns is constantly escalating over major parts around the world, causing an excess rise in water consumption due to the increase in population, variations in socioeconomic conditions, and the rise in demand of water for industrial/agricultural use [2].

The result of increase in processes for improving water quality due to the development of technology has been observed over the past years [3]. Freshwater being produced by the elimination of dissolved minerals from sea water is the process of desalination; as it proves to be an answer to the water shortage issue [4]. Thermal and membrane methods are the two main practises used for desalination [5]. Membrane methods incorporate the reverse osmosis (RO) technology where membrane fouling is sternly noticed. Multi-stage flash desalination (MSF) and multiple-effect distillation (MED) comprise of the thermal methods but high energy consumption is a challenge in these methods [6]. Vapour compression

\footnotetext{
* Corresponding author: hej246@bham.ac.uk
} 
technology (VCD) is used for small-scale applications [7]. These desalination methods have the disadvantages of high energy consumption and high operating costs [8].

In comparison to other desalination processes, significance for freeze desalination (FD) is perceived owing it has many advantages [9-14]. The salts are rejected in freeze desalination as the formation of ice prevents inclusion of any solutes. This is due to the nature of the ice crystal lattice only formed by pure water without any solutes, which is called the water solidification phenomena [9-10]. The latent heat of fusion is $335 \mathrm{~kJ} / \mathrm{kg}$ and the latent heat of vaporization is $2256.7 \mathrm{~kJ} / \mathrm{kg}$, thus indicating the low energy usage by the freeze desalination process [11-12]. A wide range of construction methods and a range of materials are acceptable in freeze desalination due to is low operating temperatures in comparison to other distillation processes that permits corrosion and scale formation [12]. Regasification of liquefied natural gas (LNG) can provide the cold energy that can be used in the freeze desalination process [14].

The salt separation technique and the salinity surge of the remaining solution after the freezing process was not studied by researchers. Therefore, in this paper, a three dimensional CFD model has been created of the formation of ice in an ice maker machine. The brine separation and the ice layer growth was also modelled using CFD where the ice in the form of pure water was generated and the brine in the remaining solution was also examined. The CFD model that has been created was then used to conduct parametric study to investigate the outcomes of freezing temperature and the geometry of the ice maker machine; on production of ice and freezing time.

\section{CFD modelling}

A single ice unit formation in an ice maker machine has been modelled using ANSYS Fluent in order to carry out computational fluid dynamics study by indirect contact freezing. Therefore as the ice unit is formed with pure water, the brine is then rejected to the rest of the solution. A combination of the modules; solidification/melting, species transport and energy (heat transfer) are used for the ice formation and the separation of brine from the ice to the rejected aqueous solution. In order to simulate the solidification process, ANSYS Fluent uses an enthalpy-porosity method. Thus, in the porous zone, the porosity value is correspondent with the liquid fraction at the liquid-solid mushy zone [15]. The key equations (1)-(4) used to model the freeze desalination process are discussed below.

Firstly, the species transport equation has been used in conjunction with the 'scheil' rule $[15,16]$ due to the fact that at micro-scale, this enables the separation of species. The diffusion of solute species in the solid is presumed to be zero when using this rule, due to the fact that as the salt water freezes, the ice (solid) formed remains as pure water. Therefore the equation for species transport is:

$$
\begin{aligned}
\frac{d}{d t}\left(\rho Y_{i, l i q}\right)+\nabla \cdot & \left(\rho\left[\beta \overrightarrow{v_{l i q}} Y_{i, l i q}+(1-\beta) Y_{i, s o l}\right]\right) \\
& =\nabla \cdot\left(\rho \beta D_{i, m, l i q} \nabla Y_{i, l i q}\right)-K_{i} Y_{i, l i q} \frac{d}{d t}(\rho(1-\beta))+\frac{d}{d t}\left(\rho(1-\beta) Y_{i, l i q}\right)
\end{aligned}
$$

Where, the liquid volume fraction is $\beta$ and $\rho$ is the density of fluid. The liquid velocity is denoted by $\overrightarrow{v_{l i q}}$. Solid and liquid mass fractions are $Y_{i, s o l}$ and $Y_{i, l i q}$ respectively. $K_{i}$ is the partition coefficient of the solute which is the mass fraction in the solid to liquid ratio at the boundary and the following equation relates these parameters: 


$$
Y_{i, \text { sol }}=K_{i} Y_{i, l i q}
$$

When using the 'scheil' rule, the dependant variable is solved using $Y_{i, l i q} . D_{i, m}$ is the mass diffusion coefficient for species in the mixture.

When solidification problems are incorporated with species transport, the energy equation becomes:

$$
\frac{d}{d t}(\rho H)+\nabla \cdot(\rho \vec{v} H)=\nabla \cdot(k \nabla T)+\frac{(1-\beta)^{2}}{\left(\beta^{2}+\varepsilon\right)} A_{m u s h} \vec{v}
$$

Where, the enthalpy is $H$, velocity is $\vec{v}$ and the mass fraction coefficient is $k$. The liquid volume fraction is $\beta$, the temperature is denoted by $T$ and the mushy zone constant is represented by $A_{\text {mus }}$. In order to avoid being divided by zero when $\beta=0$, a small number of 0.001 is given and it is represented by $\varepsilon$.

Thermal and solutal buoyancies are important factors that need to be considered when modelling freeze desalination. These buoyancies arise when there are more than one species in the mixture due to the density constantly changing with temperature and species composition. Natural convection flows are used to calculate thermal buoyancy and it develops when gravity changes the deviations in density with temperature. Consequently, as the density changes with species composition, solutal buoyancy arise and therefore the solutal buoyancy body forces are obtained from equation (4).

$$
\vec{F}_{s}=\rho_{\text {ref }} \vec{g} \sum_{i=0}^{N_{s}} \beta_{s, i}\left(Y_{l, i}-Y_{\text {ref }, i}\right)
$$

The mass fraction of the liquid phase and the reference mass fraction are represented by $Y_{l, i}$ and $Y_{\text {ref }, i}$ respectively. The gravity is denoted by $\vec{g}$, the reference density is represented by $\rho_{\text {ref }}$ and $\beta_{s, i}$ is the solutal expansion coefficient. $N_{s}$ is the total number of solute species. The combination of thermal and solutal buoyancies is the total body force. Hence, modelling thermal and solutal buoyancies allows to accurately calculate the overall solidification behaviour of salt water. Freezing of salt water is an example of such a problem where there are buoyancy induced flows in solidification when multicomponents are present. This is called the Boussinesq approach.

\section{CFD results}

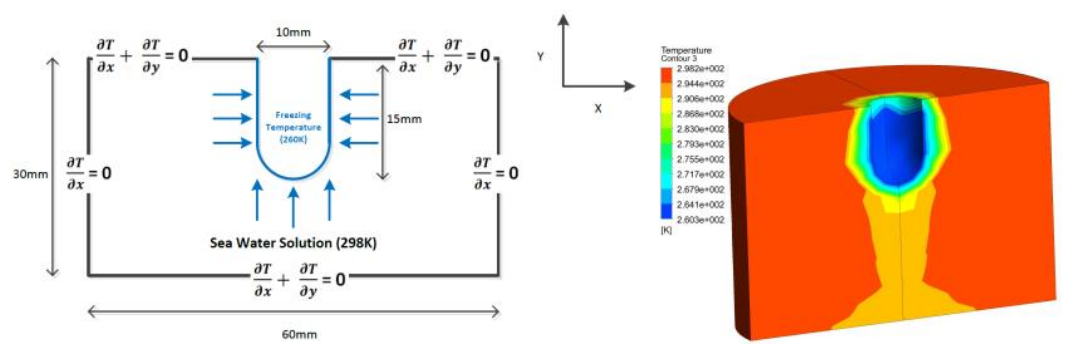

Fig. 1. (a) Schematic diagram of the problem (left) and (b) temperature contours of the developed 3D CFD model's cross sectional view (right). 
The configuration used to carry out CFD analysis is shown in figure 1(a) where a single unit of ice formation for a commercially available ice maker machine is modelled. The container holds $35 \mathrm{~g} / \mathrm{L}$ concentration of salt water with an initial temperature of $298 \mathrm{~K}$, where it has been cooled from the freezing tube (Figure 1(a)) at $260 \mathrm{~K}$ and the rest of the walls are considered to be adiabatic. The developed 3D CFD model shown in figure 1(b), is set-up with laminar incompressible flow conditions using a pressure based solver. The key modules used to simulate this process were solidification/melting and species transport. The experiment was run for 10 minutes of real time using transient analysis.

The temperature contours are shown in figure 2(a) where the solution has been cooled at a freezing temperature of $260 \mathrm{~K}$. In figure $2(\mathrm{~b})$, the distribution of temperature from the freezing location to the rest of the solution is shown after 10 minutes of freezing time. A 5.5mm thickness solid ice layer has been formed at the outer surface of the freezing tube; where the temperature at the bottom of the ice layer is $260 \mathrm{~K}$ and at the top is about $268 \mathrm{~K}$. In the ice layer formed; it is seen that the temperature is increasing from the ice layer in contact with the freezing surface, to the ice layer in contact with the rest of the solution and then it remains constant in the rest of the solution.
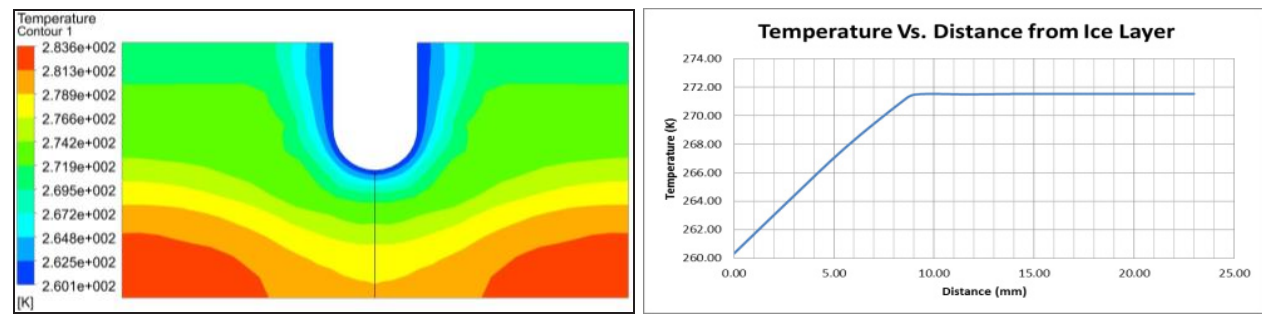

Fig. 2. (a) Temperature contours (left) and (b) temperature vs. distance from the freezing tube (right) after 10 minutes of freezing.

The solidification contours are shown in figure 3(a) where this indicated the liquid phase fraction of the solution. Minimal liquid fraction is indicated by the thick blue layer representing solid ice and the rest is the brine solution shown in red/yellow, indicating high amount of liquid fraction. In the ice layer of $5.5 \mathrm{~mm}$ thickness, the liquid fraction is zero as expected and is shown in figure 3(b).
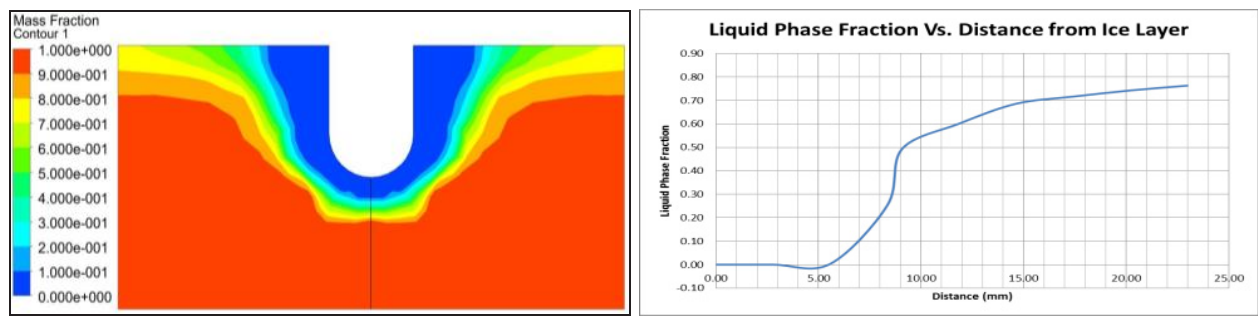

Fig. 3. (a) Liquid phase fraction contours (left) and (b) liquid phase fraction vs. distance from the freezing tube (right) after 10 minutes of freezing.

The salt water (brine) contours are shown in figure 4(a) and the graph of brine concentration versus the distance from the freezing surface is shown in figure 4(b). At the top point of the $5.5 \mathrm{~mm}$ ice layer, the brine concentration is $0.03 \%$. The brine concentration then increases away from the ice layer to about $5.9 \%$ in the rest of the solution. Therefore it is seen that the brine concentration is negligible in the ice produced and the brine salinity 
increases for the rest of the solution during the freezing process. The variations in the brine concentration in the rest of the solution are due to thermal and solutal buoyancies.
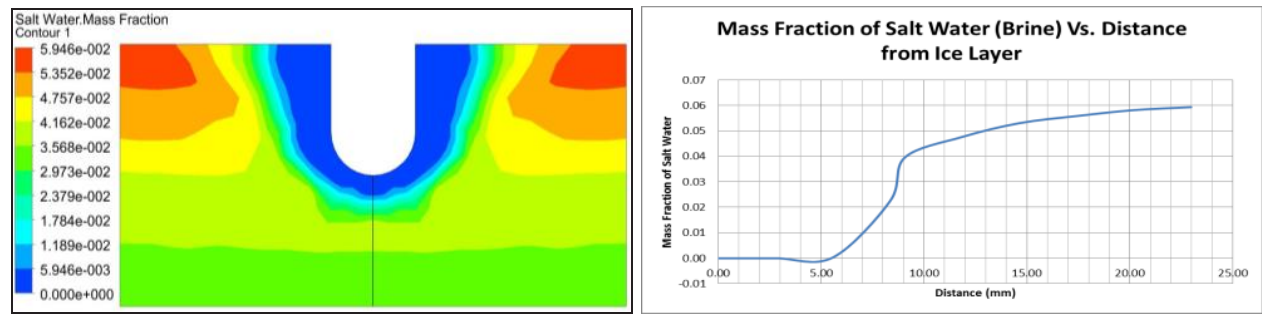

Fig. 4. (a) Salt water (brine) mass fraction contours (left) and (b) salt water (brine) mass fraction vs. distance from the freezing tube (right) after 10 minutes of freezing.

The pure water contours are shown in figure 5(a) and the graph of pure water concentration versus the distance from the freezing surface is shown in figure 5(b). Maximum concentration of pure water is present in the ice layer formed. The variations in the pure water mass fractions in the rest of the solution are due to buoyancies.
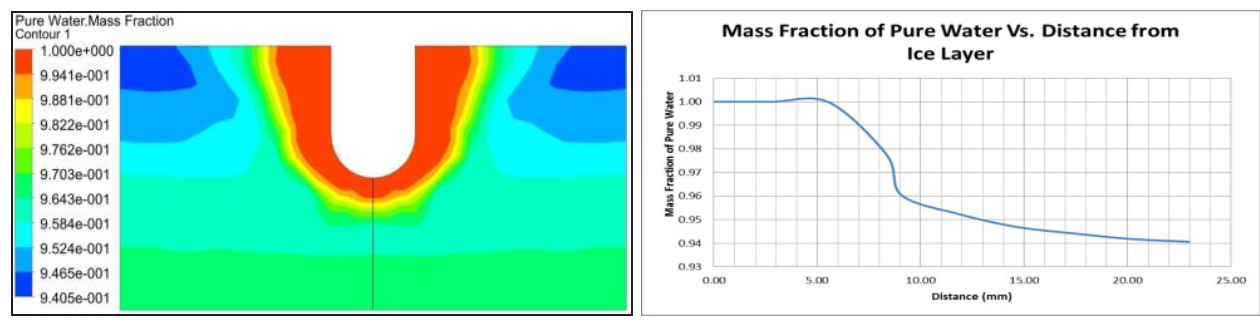

Fig. 5. (a) Pure water mass fraction contours (left) and (b) pure water mass fraction vs. distance from the freezing tube (right) after 10 minutes of freezing.

\section{Parametric analyses}

Parametric analysis has been carried out using the established CFD model to predict the effects of freezing temperature and the geometry of the ice maker machine; on ice production and freezing time.

\subsection{Freezing temperature}

Investigation of changing the freezing temperature from $230 \mathrm{~K}, 260 \mathrm{~K}$ and $270 \mathrm{~K}$ was carried out and the average solution temperature and solidification (liquid phase fraction) graphs are shown for different freezing temperatures in figure 6. It is quite apparent from figure 6(a) that when the freezing temperature is reduced, the average solution temperature decreases and allows an increased solidification rate as seen from figure 6(b). After 10 minutes of freezing; for $230 \mathrm{~K}, 260 \mathrm{~K}$ and $270 \mathrm{~K}$ of freezing temperatures, a liquid phase fraction of $0.72,0.75$ and 1 is reached respectively. This proves that lower temperatures enabled faster solidification and at $270 \mathrm{~K}$ freezing temperature, no solidification is seen after 10 minutes; due to the lower freezing point of salt water. 

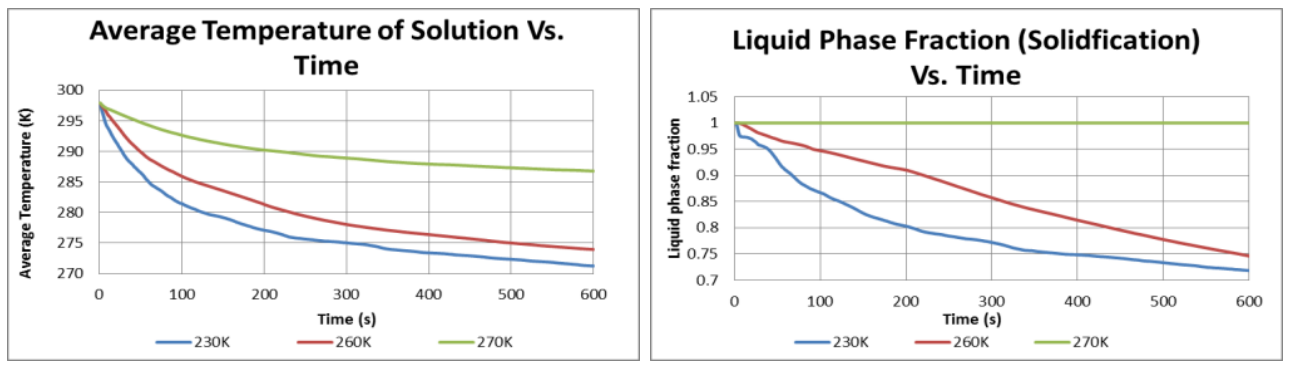

Fig. 6. (a) Average temperature of solution (left) and (b) liquid phase fraction (right) vs. time graphs for different freezing temperatures.

The temperature and the solidification (liquid phase fraction) contours for the solution are shown in figure 7 for freezing temperatures of $230 \mathrm{~K}, 260 \mathrm{~K}$ and $270 \mathrm{~K}$ after 10 minutes of real time freezing. The variations in solidification are clearly shown in the figure; where at lower freezing temperatures, more ice production can be observed. The volume of ice produced increases significantly with lower freezing temperatures, as the ice volumes at $230 \mathrm{~K}$ and $260 \mathrm{~K}$ are 5690 and $3580 \mathrm{~mm}^{3}$ respectively and no ice formation is seen with a $270 \mathrm{~K}$ freezing temperature after 10 minutes of freezing.

\section{(a) $230 \mathrm{~K}$}

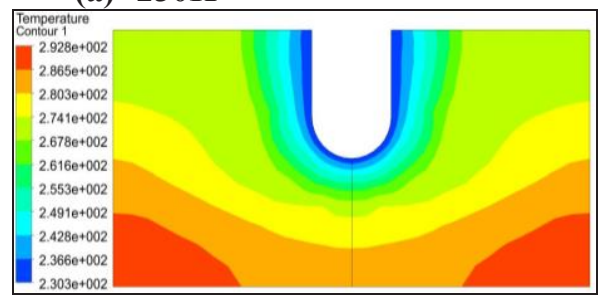

\section{(b) $260 \mathrm{~K}$}

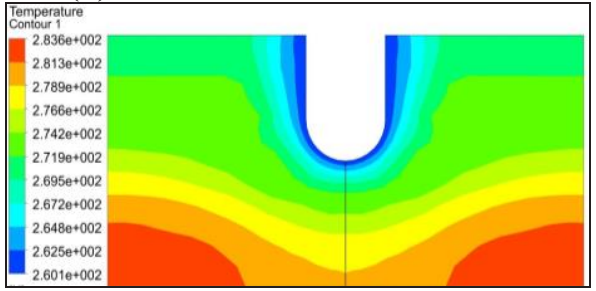

(c) $270 \mathrm{~K}$

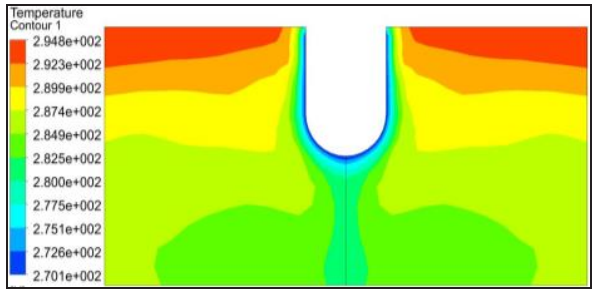

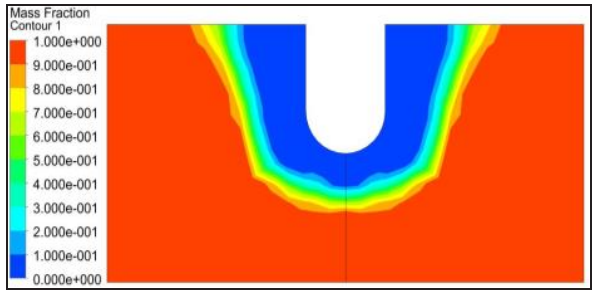
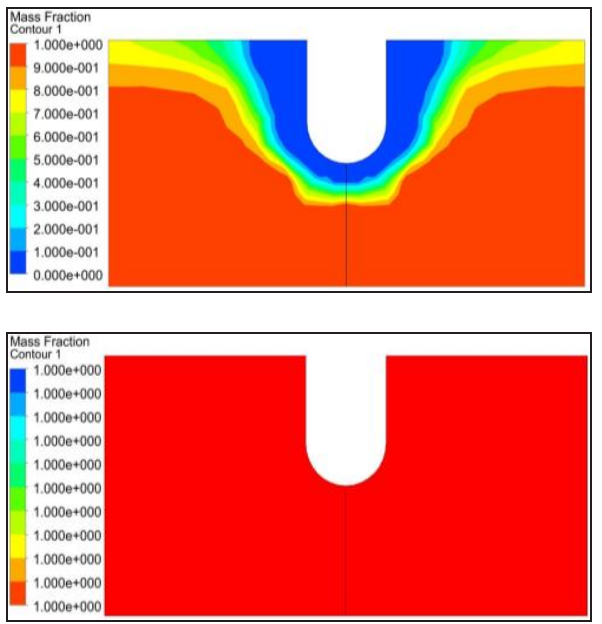

Fig. 7. Contours for temperature (left) and liquid phase fraction (right) for freezing temperatures of (a) $230 \mathrm{~K}$, (b) $260 \mathrm{~K}$ and (c) $270 \mathrm{~K}$. 


\subsection{Geometry of the ice maker machine}

The geometry of the freezing surface of a single unit has been changed to investigate the effect on ice production. The diameter and the length of the freezing tube have been varied and the solidification (liquid phase fraction) contours are shown in figure 8 . The volumes of ice produced for the freezing tube dimensions of $5 \mathrm{~mm}$ diameter is $842 \mathrm{~mm}^{3}$ and for $20 \mathrm{~mm}$ diameter is $3188 \mathrm{~mm}^{3}$. When the length of the freezing tube was $10 \mathrm{~mm}$ and $20 \mathrm{~mm}$, the ice volumes of ice produced were 1267 and $2770 \mathrm{~mm}^{3}$ respectively. Therefore it is concluded that as the diameter and the length of the freezing tube is increased, the ice production also increases due to the increase in the freezing surface area.

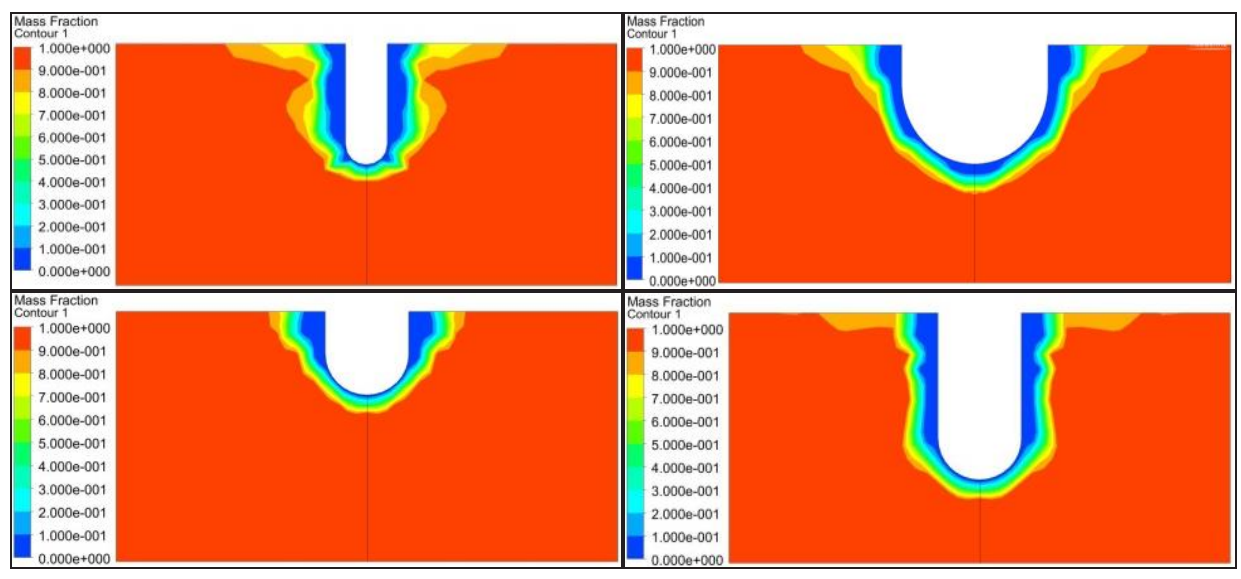

Fig. 8. Liquid phase fraction contours for (a) $5 \mathrm{~mm}$ diameter (top left), (b) 20mm diameter (top right), (c) $10 \mathrm{~mm}$ length (bottom left) and $20 \mathrm{~mm}$ length (bottom right).

\section{Conclusions}

The freeze desalination process occurs by the formation of ice as pure water; where the ice crystal lattice is formed purely of water and the salts are rejects by the nature of ice. Hence in the freeze desalination process, any solutes are prevented from entering the developing ice crystal lattice. A prodigious potential is displayed for freeze desalination due to its benefits over other desalination methods. Lower energy consumption enables freeze desalination to stand out among other desalination processes; where the latent heat of vaporisation $(2256.7 \mathrm{~kJ} / \mathrm{kg})$ is much higher than the latent heat of fusion $(333.5 \mathrm{~kJ} / \mathrm{kg})$. Another major advantage of this process is that scale formation and corrosion are diminished due to its low operating temperatures. Furthermore, regasification of liquefied natural gas (LNG) can be done to exploit the cold energy that can be used in the freeze desalination process.

Limited literature is presented for computational fluid dynamics (CFD) on freeze desalination. Hence, investigating and analysing thermodynamic processes are done by the powerful tool of CFD. A single unit of ice formation in an ice maker machine was simulated using the ANSYS Fluent software three-dimensionally. Solidification/melting, species transport and energy modules were used in constructing the CFD model.

Parametric analysis was done by expanding the established CFD model to predict the effects of freezing temperature and the geometry of the ice maker machine; on ice production and the freezing time. Hence, lower freezing temperatures allowed more ice production and faster freezing. The $230 \mathrm{~K}$ freezing temperature allowed $37.1 \%$ more ice to be produced compared to the $260 \mathrm{~K}$ freezing temperature. Additionally, increasing the 
diameter and the length of the freezing tube enabled more ice production. Thus, the geometry of $20 \mathrm{~mm}$ freezing tube diameter permitted $73.6 \%$ more ice to be produced compared to the $5 \mathrm{~mm}$ freezing tube diameter geometry. The geometry of $20 \mathrm{~mm}$ freezing tube length gave 2.19 times more ice compared to the $10 \mathrm{~mm}$ freezing tube length geometry.

The authors would like to thank the School of Mechanical Engineering, University of Birmingham, UK; for offering a PhD scholarship to carry out the research on modelling and investigation of the freeze desalination process.

\section{References}

1. M. Pedro-Monzonís, A. Solera, J. Ferrer, T. Estrela, J. Paredes-Arquiola, J. Hydrol. 527, 482-493 (2015)

2. T.I.E. Veldkamp, Y. Wada, H. de Moel, M. Kummu, S. Eisner, J.C.J.H. Aerts, P.J. Ward, Glob. Environ. Chang. 32, 18-29 (2015)

3. Y. Ramírez, L.A. Cisternas, A. Kraslawski, J. Clean. Prod. 148, 223-232 (2017)

4. H. March, WIRE. Water, 2.3, 231-243 (2015)

5. M. Rahman, M. Ahmed, X.D. Chen, Sep. Purif. Rev. 35, 2, 59-96, (2006)

6. J. Chang, J. Zuo, K.J. Lu, T.S. Chung, Water Res. 102, 282-293 (2016)

7. M.C. Fragkou, J. McEvoy, Desalination 397, 1-8 (2016)

8. A. Chafidz, E.D. Kerme, I. Wazeer, Y. Khalid, A. Ajbar, S.M. Al-Zahrani, J. Clean. Prod. 133, 631-647 (2016)

9. D.G. Randall, J. Nathoo, J. Water Process Eng. 8, 186-194 (2015)

10. R. Fujioka, L.P. Wang, G. Dodbiba, T. Fujita, Desalination 319, 33-37 (2013)

11. A.A.A. Attia, Desalination 254, 1-3, 179-184 (2010)

12. P.M. Williams, M. Ahmad, B.S. Connolly, D.L. Oatley-Radcliffe, Desalination 356, 314-327 (2015)

13. Z. Lu, L. Xu, DESWARE, 2 (2014)

14. W. Cao, C. Beggs, I.M. Mujtaba, Desalination 355, 22-32 (2015)

15. ANSYS Fluent Theory Guide. 15th ed. USA: ANSYS, Inc. (2013)

16. V.R. Voller, D. Brent, C. Prakash, Int. J. Heat Mass Transfer 32, 9, 1719-1731 (1989) 